\title{
Factores de riesgo asociados a caries y enfermedad periodontal en Centro de Educación
} Básica Especial de Huánuco, 2017

\section{Risk factors associated with caries and periodontal disease in a Center of Special Basic Education in Huánuco, 2017}

Yulith Basilio-Bernardo ${ }^{1}$, Karina P. Cavalié-Martel ${ }^{1 *}$, Marisol R. Ortega-Buitron ${ }^{1}$

\begin{abstract}
Resumen
Objetivos. Identificar factores de riesgo asociados a caries y enfermedad periodontal en niños de 718 años de edad en Centro de Educación Básica Especial "Niño Jesús de Praga" Huánuco 2017. Materiales y métodos. Diseño Observacional, Analítico de casos y controles. Empleándose ficha de recolección tipo encuesta. Muestra de 60 niños especiales (grupo de estudio) y 60 niños no especiales (grupo control). Variable dependiente: caries y enfermedad periodontal (con etiologías más frecuentes en cavidad oral). Variables independientes: índice de higiene oral, tiempo de parto, complicación durante la gestación, Ph salival, ingesta de carbohidratos y tipo de parto. Resultados Entre los factores de riesgo para caries y enfermedad periodontal, no existe diferencia estadísticamente significativa $(p<0,05)$ con el tiempo de parto, ni los otros factores Se determinó como factores de riesgo para caries: Índice de higiene oral, tiempo de parto y complicación durante la gestación; y factores de riesgo para la Enfermedad periodontal: $\mathrm{Ph}$ salival, ingesta de carbohidratos y tipo de parto. Además, no se encontró diferencias significativas $(p>0,05)$ entre los Índices CPOD+ceod para caries ( $p: 0,36)$ e índice INTPC para enfermedad periodontal $(p: 0,89)$ para ambos grupos. Conclusiones. Encontramos diferencia significativa entre el factor Tiempo de parto y la caries y enfermedad periodontal que afectan a ambos grupos. Se encontró factores de riesgo específicamente para caries y enfermedad periodontal que no son tan usados para estas enfermedades bucales en ambos grupos y no encontrándose diferencia significativa entre los índices usados.
\end{abstract}

Palabras clave: caries dental, enfermedades periodontales, factores de riesgo (DeCS fuente Bireme)

\begin{abstract}
Objectives: To identify the risk factors associated with caries and periodontal disease in children from 7to18 years of age in the Center of Special Basic Education "Niño Jesús de Praga" in Huánuco 2017. Materials and methods: Observational, Analytical Design of cases and controls. A surveytype collection sheet was used. The sample was constituted of 60 special children (study group) and 60 non-special children (control group). Dependent variable: caries and periodontal disease (because they are the most frequent disease in the oral cavity). Independent variables: oral hygiene index, labor time, complication during pregnancy, salivary $\mathrm{pH}$, carbohydrate intake and type of labor Results: Among the risk factors for caries and periodontal disease, there is no statistically significant difference $(p<0.05)$ with the labor time, nor the other factors. The following factors were determined as risk factors for caries: Oral hygiene index, labor time and complications during the pregnancy; and risk factors for periodontal disease: salivary $\mathrm{pH}$, carbohydrate intake and type of labor. Furthermore, no significant differences $(p>0.05)$ were found between the DMFT+ dft index for caries ( $p: 0.36)$ and the CPITN index for periodontal disease ( $p: 0.89)$ for both groups. Conclusions: We found a significant difference between labor time and caries and periodontal disease that affect both groups. The risk factors were found specifically for caries and periodontal disease that are not as widely used for these oral diseases in both groups and no significant difference was found between the used indexes.
\end{abstract}

Keywords: dental caries, periodontal diseases, risk factors (MeSH source NLM).
Universidad Nacional Hermilio Valdizán, Huánuco, Perú

ORCID:

https://orcid.org/0000-0003-4252-8893

\section{Correspondencia a:}

Karina Paola Cavalié Martel

Dirección: Av. Universitaria 600-601 Pillcomarca, Huánuco, Perú

Email:pao1405@yahoo.es

Fecha de recepción: 21 de octubre de 2019

Fecha de aprobación: 03 de abril de Fecha
2020

Citar como: Basilio-Bernardo Y, CavaliéMartel K, Ortega-Buitron M. Factores de riesgo asociados a caries y enfermedad periodontal en Centro de Educación periodontal en Centro de Educación Peru. Investig. Salud. [Internet]; 4(2): 7076. Available from:

http://revistas.unheval.edu.pe/index.ph $\mathrm{p} / \mathrm{repis} /$ article/view/502
2616-6097/@2020. Revista Peruana de Investigación en Salud. Este es un artículo Open Access bajo la licencia Permite copiar y redistribuir el material en culatovier medio o formato. Usted debe dar crédito de manera adecuada, brindar un enlace a la licencia, e indicar si se han realizado cambios.

\section{Introducción}

La Organización Mundial de la Salud precisó que se debe detectar y atender tempranamente a los niños con deficiencias o alteraciones del desarrollo (1), concluyendo que la multicausalidad de la discapacidad y la complejidad de los diversos factores que interactúan para producir discapacidad, nos empujan a la búsqueda e identificación de enfoques y estrategias que permitan dar respuestas integrales insertadas en los programas y políticas públicas sobre atención a las personas con discapacidad, su rehabilitación e inserción social, especialmente transversalizando el tema en todas las iniciativas de desarrollo que conduzcan al bienestar humano (2). El Ministerio de Salud aún no cuenta con registros médicos oficiales sobre cuál es la enfermedad bucal más prevalente en niños especiales con respecto a los factores de riesgo para caries y enfermedad periodontal.

Shapira et al.(3) realizo un estudio en población Israelí con niños de edades de 11 años y adultos con edad promedio de 22 años en los cuales encontró que los adultos especiales tuvieron problemas periodontales severos que incluso requirieron cirugía periodontal y su 
promedio de dientes cariados, perdidos y obturados fue bastante menor que la población adulta normal; en el caso de los niños especiales estos mostraron un índice de caries similar a la población no especial y necesidad de tratamiento periodontal.

El estudio hecho en Bélgica a niños especiales encontró que no existe diferencia significativa en la experiencia de caries con la población normal (4).

Un trabajo realizado sobre patología bucal prevalente en niños excepcionales de Venezuela, descubrieron que los niños especiales tienen una alta prevalencia de caries y maloclusiones en comparación con otras patologías como Síndrome Down, Sordomudos, Parálisis Cerebral y solo fue superado por los ciegos (5). Con lo cual concluyeron que es indispensable tomar acciones de salud bucal ante estos problemas en los grupos de mayor riesgo como son los autistas y los ciegos a fin de dar una solución eficaz a sus patologías bucales y mejorar así su calidad de vida.

Beck (6) manifiesta que un factor de riesgo es un factor ambiental, conductual o biológico confirmado por una secuencia temporal la cual si se presenta directamente incrementa la probabilidad que la enfermedad ocurra y en su ausencia reduce esta probabilidad.

Jenkins y Papapanou (7) encontraron que la gingivitis es la afección más común, su prevalencia, severidad y extensión aumentan con la edad, comenzando en la dentición primaria, hasta alcanzar su apogeo en la pubertad seguido de un pequeño descenso en la adolescencia. La limitada información existente con respecto a la periodontitis en la dentición primaria apunta a una prevalencia en niños de origen europeo de aproximadamente del $5 \%$. Generalmente son pocos los sitios que son afectados y la cantidad de perdida de unión es inconsecuente (8).

La caries dental y enfermedad periodontal son procesos patológicos complejos de origen infeccioso y transmisible que afecta a las estructuras blandas y dentarias que se caracteriza por un desequilibrio bioquímico: que produce cavitación y alteraciones del complejo dentino pulpar (9).

Nuestra investigación se enmarca en sentar las bases para identificar el comportamiento de estos factores de riesgo que conllevan a estas enfermedades orales en este grupo humano con necesidades especiales que en la actualidad tiene diversas controversias incluso en el mismo diagnóstico médico el cual para muchos investigadores está en vías de esclarecerse.

Según evidencia científica estos pacientes presentan un elevado riesgo de caries dental y de enfermedad periodontal sobre todo en la edad infantil, debido a la referencia que tienen éstos por el consumo de alimentos pegajosos y azucarados; que adicionado a la deficiente higiene oral, las alteraciones de conducta, deficiencias gastrointestinales como: maladigestión, malaabsorción , reflujo esofágico, deficiencias enzimáticas, gastritis, deficiencias metabólicas junto a las deficiencias motoras e inmunitarias, sobre todo a nivel de los linfocitos; propias de la enfermedad, hacen que el niño especial sea de especial cuidado para el tratamiento odontológico siendo la prevención el camino de elección a la hora de tratar a este tipo de pacientes (9). Era necesario realizar la investigación en el lugar de los hechos para que la investigación tenga confiabilidad y consistencia en los datos expresados. Por lo tanto, el Objetivo de la presente investigación fue Identificar los factores de riesgo que ocasionan caries dental y enfermedad periodontal en niños de 7 a 18 años del Centro de Educación Básica Especial “Niño Jesús de Praga” de Huánuco 2017.

\section{Materiales y Métodos}

\section{Tipo de estudio}

El tipo del estudio fue cuantitativo, diseño observacional, analítico de casos y controles, porque se comparó a un grupo de niños con características especiales (estudio o casos) con un grupo de niños que no presentan estas características (controles o testigos).

\section{Variables de estudio}

La variables estudiadas son: Variable dependiente: Caries dental y Enfermedad periodontal (etiologías más frecuentes en la cavidad oral asociadas a la acumulación de placa bacteriana). Variable independiente: Factores de Riesgo para enfermedades de caries y enfermedad periodontal (como son índice de higiene oral (IHO), tiempo de parto, complicación durante la gestación, Ph salival, 
ingesta de carbohi-dratos y tipo de parto).

\section{Población y muestreo}

La población fueron todos los alumnos del Centro de Educación Básico Especial "NIÑO JESUS DE PRAGA", haciendo un total de 140 niños. El tamaño de la muestra fue de 120 niños. El cálculo de la muestra fue por el tipo de muestreo no probabilístico por conveniencia a fin de que no intervengan factores externos. Por tanto la muestra estuvo conformada por los niños especiales entre 7 a 18 años, agrupados en dos grupos, 60 niños especiales para el grupo de estudio y 60 niños no especiales para el grupo control.

\section{Procedimientos}

Los niños seleccionados tenían un diagnóstico médico psiquiátrico de padecer el trastorno y además tenían hermanos dentro de las edades del estudio.

Para el procedimiento de estudio se reunió al grupo de niños especiales y niños no especiales un día y hora prestablecidas en el Centro de Educación Básica Especial "Niño Jesús de Praga". La ficha fue Ilenada empezando por los datos de filiación que fueron consultados con el padre o tutor de cada niño. Para la recolección de datos índices de caries dental y enfermedad periodontal se empleó:

- Índice de caries dental (CPOD+ceod) para dientes primarios y permanentes.

- Índice de necesidades de tratamiento periodontal comunitario (INTPC).

- Parámetros para la evaluación de Factores de riesgo para caries y enfermedad periodontal.

\section{Análisis de datos}

Para el procesamiento de los datos se realizó un análisis de datos estadísticos de tipo inferencial utilizando la prueba de Chi Cuadrado $\left(\mathrm{x}^{2}\right)$ mediante el programa estadístico SPSS 15 para poder tabular los datos estadísticos provenientes de los resultados del estudio. Estos resultados fueron agrupados en tablas de contingencia y se vio su relación con los índices estudiados (CPOD+ceod; INTPC) y luego se utilizó el paquete estadístico EPIDAT versión 3.1 para establecer los niveles de riesgo que ejercen cada uno de los factores sobre los índices. Para la determinación del riesgo se realizó con el meta análisis de ODDS RATIO y todos los factores de riesgo utilizando el programa estadístico EPIDAT versión 3.1. Para la determinación de las diferencias entre ambas poblaciones se utilizó la prueba Chi Cuadrado $(p<0,05)$ mediante el programa estadístico SPSS 15.

\section{Resultados}

Tabla 1 y 2. Índice de Caries Dental (CPOD + ceod) en grupo de niños especiales y grupo de niños no especiales.

Se establecieron dos bases de datos con respecto a CPOD+ceod (índice de caries dental) para ambos grupos, grupo de niños especiales (grupo en estudio) y grupo de niños no especiales (grupo control).

Tabla 1. Índice de Caries Dental (CPOD+ceod) en grupo de niños especiales (GE)

\begin{tabular}{lcccc}
\hline \multicolumn{1}{c}{ Factor } & A & B & C & D \\
\hline IHO & 41 & 8 & 8 & 3 \\
$\begin{array}{l}\text { Tiempo de } \\
\text { parto }\end{array}$ & 11 & 3 & 38 & 8 \\
$\begin{array}{l}\text { Complicación } \\
\text { en gestación }\end{array}$ & 27 & 11 & 11 & 11 \\
$\begin{array}{l}\text { Ph saliva } \\
\text { Ingesta de }\end{array}$ & 24 & 3 & 30 & 3 \\
carbohidratos & 34 & 8 & 15 & 3 \\
Tipo de parto & 15 & 3 & 34 & 8 \\
\hline
\end{tabular}

Tabla 2. Índice de Caries Dental (CPOD+ceod) en grupo de niños no especiales (GC)

\begin{tabular}{lcccc}
\hline \multicolumn{1}{c}{ Factor } & A & B & C & D \\
\hline IHO & 20 & 3 & 26 & 11 \\
Tiempo de & 8 & 38 & 3 & 11 \\
parto & & & & \\
$\begin{array}{l}\text { Complicación } \\
\text { en gestación }\end{array}$ & 8 & 3 & 34 & 15 \\
Ph saliva & 11 & 8 & 33 & 8 \\
Ingesta de & 34 & 15 & 8 & 3 \\
carbohidratos & & & 15 & 11 \\
\hline Tipo de parto & 31 & 3 &
\end{tabular}

$\mathrm{A}, \mathrm{B}, \mathrm{C}$ y $\mathrm{D}$ es el $\mathrm{N}^{\circ}$ de individuos con CPOD + ceod alto o bajo según el factor $\mathrm{IHO}$ y Factores de Riesgo referente al Grupo de niños especiales (grupo en estudio) y no especiales (grupo control). 
Tabla 3. Comparación de las frecuencias del Índice de Enfermedad Periodontal (INTPC) en grupo de niños especiales y grupo de niños no especiales

\begin{tabular}{|c|c|c|c|c|c|c|c|c|}
\hline \multirow{3}{*}{ Grupo } & \multicolumn{6}{|c|}{ INTPC } & \multirow{2}{*}{\multicolumn{2}{|c|}{ Total }} \\
\hline & \multicolumn{2}{|c|}{0} & \multicolumn{2}{|c|}{1} & \multicolumn{2}{|c|}{2} & & \\
\hline & $\mathrm{N}^{\circ}$ & $\%$ & $\mathrm{~N}^{\circ}$ & $\%$ & $\mathrm{~N}^{\circ}$ & $\%$ & $\mathrm{~N}^{\circ}$ & $\%$ \\
\hline Especiales & 39 & 65,0 & 17 & 28,3 & 4 & 6,7 & 60 & 100 \\
\hline No especiales & 40 & 66,7 & 0 & 0,0 & 20 & 33,3 & 60 & 100 \\
\hline
\end{tabular}

En la tabla 3, se observa que en el grupo de niños especiales el $65 \%$ tuvo valor 0 y el $28,3 \%$ valor 1 . En el caso de los niños no especiales el $66,7 \%$ tuvo valor 0 en comparación con el $33,3 \%$ que tuvo valor de 2 .

Tabla 4 y 5. Índice de Enfermedad Periodontal (INTPC) en grupo de niños especiales y grupo de niños no especiales.

Se establecieron dos bases de datos con respecto a INTPC (índice de enfermedad periodontal) para para ambos grupos, grupo de niños especiales (grupo en estudio) y grupo de niños no especiales (grupo control).

Tabla 4. INTPC en Grupo de Niños Especiales (GE)

\begin{tabular}{lcccc}
\hline \multicolumn{1}{c}{ Factor } & A & B & C & D \\
\hline $\mathrm{IHO}$ & 16 & 34 & 7 & 3 \\
Tiempo de & 3 & 8 & 9 & 30 \\
$\begin{array}{l}\text { parto } \\
\text { Complicación }\end{array}$ & 11 & 27 & 11 & 1 \\
en gestación & 15 & 11 & 7 & 27 \\
$\begin{array}{l}\text { Ph saliva } \\
\text { Ingesta de }\end{array}$ & 19 & 27 & 3 & 11 \\
carbohidratos & 11 & 8 & 11 & 30 \\
\hline Tipo de parto & 11 &
\end{tabular}

Tabla 5. INTPC en Grupo de Niños No Especiales (GC)

\begin{tabular}{lcccc}
\hline \multicolumn{1}{c}{ Factor } & A & B & C & D \\
\hline IHO & 8 & 11 & 11 & 30 \\
Tiempo de & 3 & 3 & 16 & 38 \\
parto & & & & \\
$\begin{array}{l}\text { Complicación } \\
\text { en gestación }\end{array}$ & 3 & 3 & 16 & 38 \\
Ph saliva & 8 & 11 & 11 & 30 \\
Ingesta de & 11 & 38 & 8 & 3 \\
carbohidratos & 16 & 18 & 3 & 23 \\
Tipo de parto & 16 & & & \\
\hline
\end{tabular}

$A, B, C$ y $D$ es el $N^{\circ}$ de individuos con INTPC alto o bajo según el factor IHO y Factores de Riesgo referente al Grupo de Niños Especiales (G.E) y No Especiales (G.C).

Tablas 6 y 7. Valores Odds Ratio (OR) y Determinación de Factores de Riesgo de Caries dental y Enfermedad Periodontal en Niños Especiales.

Se puede observar que según la Teoría del Riesgo que se usó para la determinación de los factores de riesgo para caries dental y enfermedad periodontal en niños especiales se tuvo en cuenta todos los factores considerados en la operacionalización de las variables. Cuando se intentó establecer si existían diferencias significativas entre ambas poblaciones de niños especiales y no especiales con respecto a sus factores de riesgo se encontró que solo el Factor tiempo de parto fue el único que fue estadísticamente significativo al $95 \%$ de confiabilidad $(0,03)$, para ello se utilizó el Programa estadístico SPSS versión 15 y se usó la prueba de Chi cuadrado.

Tabla 6: Valores Odds Ratio (OR) para los Factores de Riesgo de Caries Dental y Enfermedad Periodontal en Niños Especiales

\begin{tabular}{|c|c|c|}
\hline Factor & $\begin{array}{c}\text { Caries } \\
\text { (OR) }\end{array}$ & $\begin{array}{c}\text { Enf. Periodontal } \\
\text { (OR) }\end{array}$ \\
\hline $\mathrm{IHO}$ & 2,75 & 0,22 \\
\hline $\begin{array}{l}\text { Tiempo de } \\
\text { parto }\end{array}$ & 0,60 & 0,80 \\
\hline $\begin{array}{l}\text { Complicación } \\
\text { en gestación }\end{array}$ & 2,33 & 0,42 \\
\hline $\mathrm{Ph}$ salival & 0,75 & 4,66 \\
\hline $\begin{array}{l}\text { Ingesta de } \\
\text { carbohidratos }\end{array}$ & 1,12 & 2,14 \\
\hline Tipo de parto & 0,88 & 4,00 \\
\hline
\end{tabular}


Tabla 7. Determinación de los Factores de Riesgo para Caries Dental y Enfermedad Periodontal de Niños Especiales

\begin{tabular}{lcc}
\hline \multicolumn{1}{c}{ Factor } & $\begin{array}{c}\text { Caries } \\
\text { (OR) }\end{array}$ & $\begin{array}{c}\text { Enf. Periodontal } \\
\text { (OR) }\end{array}$ \\
\hline IHO & Riesgo & - \\
Tiempo de & - & - \\
parto & - & - \\
Complicación & Riesgo & Riesgo \\
gestación & - & Riesgo \\
Ph saliva & - & Riesgo \\
Ingesta \\
carbohidratos
\end{tabular}

Tabla 8. Análisis inferencial entre factores de riesgo con Caries Dental y Enfermedad Periodontal de Niños Especiales

\begin{tabular}{lcc}
\hline \multicolumn{1}{c}{$\begin{array}{c}\text { Factor de } \\
\text { riesgo }\end{array}$} & $\mathbf{X}^{2}$ & $\mathbf{p}$ \\
\hline $\begin{array}{l}\text { Tiempo de } \\
\text { parto }\end{array}$ & 4,62 & 0,03 \\
$\begin{array}{l}\text { Índice de } \\
\text { CPOD }\end{array}$ & 67,47 & 0,36 \\
$\begin{array}{l}\text { Índice de } \\
\text { INTPC }\end{array}$ & 0,0194 & 0,89 \\
\hline
\end{tabular}

\section{Discusión}

Los pacientes especiales se consideran, según la Academia Americana de Psiquiatría en su Manual de Diagnóstico y Estadística como un desorden neuropsiquiatrico profundo del desarrollo que implica un desorden orgánico caracterizado por anormalidades en el cerebro especialmente en el sistema límbico y cerebelar. Siendo el Objeto de la presente investigación establecer cuáles eran los factores de riesgo para caries y enfermedad periodontal que presentan los pacientes especiales y no especiales (10). El Centro de Educación Básica especial de Huánuco, es un centro de educación para personas con discapacidad mental y neurológica que abarca un número bastante grande de la población especial de Huánuco en total 140 estudiantes de los cuales solo 60 cumplían con la condición de tener hermanos. Esta condición actualmente es poco frecuente debido a que ahora los padres de estos niños saben que existe una alta probabilidad genética de que los hermanos de estos niños tengan problemas similares en el área de la comunicación o del aprendizaje, como lo sugieren los estudios de Folstein y Rutter que determinaron en estudios hechos tanto en hermanos como en gemelos fraternos e idénticos de niños especiales, que la condición tiene una fuerte predisposición de transmisión genética hacia los hermanos que pueden sufrir déficits cognitivos y sociales (11). Por otro lado aunque la caries no es hereditaria existe la resistencia contra la caries dental que es el resultado de la combinación de varios factores hereditarios (12). Por ello se decidió que se hiciera esta investigación en niños especiales y sus hermanos sanos (es decir aquellos que no tenían alteraciones de fondo) a fin de establecer un patrón de comportamiento de los factores de riesgo para caries y enfermedad periodontal más controlado y de tener la menor intervención de factores extraños a los objetivos de la presente investigación.

Dentro de los datos observados en la presente investigación se encontró que en el manejo de la conducta de los niños especiales en el examen dental, este dato mostró que un $56,7 \%$ de los niños especiales fueron colaboradores y un $43,3 \%$ no lo fueron, estos hallazgos resultan interesantes considerando lo que Morinushi afirma que muchos profesionales odontólogos han notado la dificultad en el manejo de conducta de los niños especiales durante el tratamiento dental, además describen el uso de medicación para el manejo de conducta de estos niños. Sin embargo los niños especiales del presente estudio sí pudieron ser manejados sin farmacología alguna con resultados aceptables (13).

Otro dato interesante del estudio fue el grado de instrucción de las madres de los niños especiales, encontrándose que el mayor porcentaje lo obtuvo el rubro de educación superior $(45.0 \%)$ lo cual concuerda con los datos hallados en otras investigaciones como las de Bowley y Gardner afirman que los padres de estos pacientes suelen tener altos niveles de educación y de nivel social, sin embargo recientes estudios afirman que en todos los niveles sociales y culturales se produce en la misma proporción (14).

Con respecto a la presencia de complicaciones durante la gestación, se encontró en términos generales que un $40 \%$ de los niños especiales no presentaron complicaciones y un $60 \%$ si tuvieron complicaciones en comparación con los hermanos no especiales 
en las cuales el $86,7 \%$ no presentaron complicaciones durante la gestación.

Asimismo en el factor de IHO, se encontró que fue de riesgo para la presencia de caries dental en niños especiales (OR: 2,75) y en no especiales (OR: 2,14) siendo ligeramente mayor en los niños especiales. Este hallazgo coincide con lo reportado en la literatura debido a que numerosos estudios afirman que la mala higiene bucal es un factor de riesgo significativo en la presencia de caries dental, por otra parte la edad de la placa bacteriana tiene un efecto significativo sobre la producción de ácidos. Las placas bacterianas maduras tienen mayor habilidad para disminuir el $\mathrm{Ph}$ a partir de soluciones de azúcar que las placas recientemente formadas (15). Por otra parte este factor no mostró una influencia significativa con respecto a la presencia de enfermedad periodontal en especiales y no especiales (16).

Con respecto al tipo de parto los niños especiales tuvieron un mayor porcentaje de parto normal $(66,7 \%)$ en comparación con los no especiales $(33,3 \%)$ no encontrándose diferencias estadísticamente significativas, y se determinó que este era un factor de riesgo para enfermedad periodontal en ambas poblaciones (especiales OR: 4,00; no especiales OR: 4,80 ) además se encontró que también fue un factor de riesgo de caries en no especiales (OR: 6,00). Con respecto a este factor se sabe que en la primera infancia, durante el parto vaginal el bebé antes de nacer recoge los lactobacillus de la vagina materna y se alojan en la boca del bebe y se mantienen por 6 días, hasta que son eliminados naturalmente (17).

Por lo encontrado en el presente estudio se comprueba que existen diferencias estadísticamente significativas $(P<0,05)$ solo en uno de los factores de riesgo que constituyó el tiempo de parto, lo que indica que aunque los demás factores de riesgo encontrados para caries dental en niños especiales como son: índice de higiene oral (IHO), y complicación durante la gestación; además de los factores de riesgo para enfermedad periodontal como son: ph salival, ingesta de carbohidratos; son factores comprobados de riesgo, no existen diferencias estadísticamente significativas entre ambas poblaciones estudiadas. Por lo que los programas preventivos enfocados hacia este tipo de grupo poblacional no variaran mucho en cuanto a contenido pero si en cuanto al enfoque del receptor debido a que no solo se entrenará al paciente especial sino también a todo el entorno familiar, concluyéndose que aunque no existen mayoritariamente diferencias estadísticamente significativas entre la prevalencia de los factores de caries y enfermedad periodontal, ni entre la prevalencia de caries y enfermedad periodontal, entre los niños especiales del centro de Educación Básica especial de Huánuco y sus hermanos no especiales, no significa que no existan estas enfermedades en ellos, incluso el hecho de que se haya estudiado a los niños especiales y sus hermanos es debido al fuerte patrón genético que presentan (18).

Por lo menos esto es lo que se ha encontrado en la presente investigación que haría pensar que los factores de riesgo para ambas enfermedades bucales, en realidad son diferentes en cada población y los encontrados en este estudio corresponden a niños especiales de la población huanuqueña y de sus hermanos sanos no especiales (19). Entonces, aunque ambas poblaciones quizás presentan factores de riesgo similares, existe la posibilidad que, con una adecuada educación en cuanto a las medidas preventivas de salud oral y el cuidado necesario para mantener un estado de salud bucal en estos niños especiales, se puedan lograr resultados prometedores en cuanto a la prevención de enfermedades bucales en esta población, que harían mucho más llevadera la condición de vida de estos pacientes con discapacidad.

\section{Conclusión}

Los factores de riesgo que determinan la presencia de caries y enfermedad periodontal en la población de niños especiales están asociados a IHO, tiempo de parto, complicación durante la gestación, Ph salival, Ingesta de carbohidratos y tipo de parto. Siendo el tiempo de parto el único que mostró diferencias estadísticamente significativas.

\section{Agradecimiento}

A los niños especiales del Centro de Educación Básica Especial "Niño Jesús de Praga" de Huánuco. 
1. OMS. Informe salud en el mundo [internet]. 2006 [consultado15 de feb 2006]; 34(1): 68. Disponible en : https://www.who.int/whr/2006/es/

2. De la Roca Arias WJ. Prevalencia de enfermedad periodontal en pacientes con Síndrome de Down. [Tesis de Cirujano Dentista]. Lima: Universidad Inca Garcilaso de la Vega; 2016.

3. Shapira J, Mann J, Tamari I. Oral health status and dental needs of an autistic population of children and young adults. Spec Care Dentist [internet].1989 [consultado Mar-Apr 1989]; 9(2): 38-41.

4. Gilberto A. Perfil de salud bucal enfermedad bucal en los escolares de 6, 12

y 15 años de la provincia de Carhuaz, región Ancash, 2016. Rev. 2017; 4(2): 519526. Disponible en:

http://revistas.uladech.edu.pe/index.php/in crescendoalud/article/view/1790/1420

5. Gómez S, Doreste J, Sierra A. Estado periodontal de los escolares de 7 a 12 años de edad en Canarias. Revista del llustre Consejo general de Odontología y Estomatología de España.3 (8). Disponible en http// medigraphic.com. Consultado 6 abril 2015.

6. Beck A, Beyond T. A theory of modes, personality, and psychopathology. 1996; 35(1): 1-25.

7. Jenkins W, Papapanou P. Epidemiology of periodontal disease in children and adolescents. Periodontology. 2000; 26(1): 16-32.

8. Marchena P. Relación entre el nivel de conocimiento sobre salud bucal de los padres y el índice de higiene oral en niños con habilidades diferentes del centro de educación básica especial la Victoria2015. Universidad Señor de Sipan. Facultad de Ciencias de la Salud. Académico Profesional de Estomatología. Tesis para obtener grado de cirujano dentista. 2015.

9. Yirina P, Baonelys T, Arelis B. Factores de riesgo de periodontopatías. Rev. 2015; 19(2). Disponible en:

http://scielo.sld.cu/scielo.php?script=sci_a rttext\&pid=S1560-3812015000200009
10. Perdomo B, Torres D, Paredes Y. Síndrome de Down e higiene bucal: Lineamientos para padres, cuidadores y docentes de investigación Odontológica Revista venezolana de investigación odontológica. 2014; 2 (2): 156-169.

11. Folstein S, Rutter M. Infantile autism a genetic study of 21 twin pairs. J Child Psychol Psychiatry. 1977; 18(4): 297-321.

12. Tirado A, Díaz C, Ramos M. Salud bucal en escolares con síndrome de Down en Cartagena (Colombia). Revista Clínica de Medicina de Familia.2015; 8(2): 110-118.

13. Morinushi T, Ueda Y, Tanaka C. Autistic children: experience and severity of dental caries between 1980 and 1995 in Kagoshima City, Japan. Journal of Clinical Pediatric Dentistry: july 2001; 25 (4): 323328.

14. Bowley A, Gardner L. El niño minusvalido: Guía educativa y psicológica para el minusvalido organico. Editorial continental 1985; 2(6): 221-235.

15. Toledo B, López M, Yamamoto N. Enfermedad periodontal en pacientes adolescentes con síndrome de Down. Presentación de caso. Revista odontológica mexicana. 2014; 18(3): 191-198.

16. Motta A. Higiene bucal y gingivitis en alumnos con discapacidades intelectuales del Colegio Especial "Santa Teresa de Courdec" mayo-junio 2011 [Tesis para optar el grado de Cirujano Dentista]. Lima, Perú: Universidad San Martin de Porres; 2014.

17. Kutsch, V. Caries dental: un modelo médico actualizado de evaluación de riesgos. Pub. Med. 2014; 111(4): 280-5.

18. Opal S, Garg S, Jain J, Walia. Factores genéticos que afectan el riesgo de caries dental. PubMed. 2015; 60 (1): 2-11.

19. Rodríguez A, León M, Arada A, Martínez M. Factores de riesgo y enfermedades bucales en gestantes. Revista de Ciencias Médicas. 2013; 17 (5): 51-63 